\title{
Área de actividad y movimientos de Liomys irroratus (Rodentia: Heteromyidae) en una selva mediana de Tuxtepec, Oaxaca, México
}

\section{Activity area and movements of Liomys irroratus (Rodentia: Heteromyidae) in semideciduos tropical forest of Tuxtepec, Oaxaca, Mexico}

\author{
Antonio Santos-Moreno ${ }^{\varpi}$ y Aremi E. Santiago-Marcial \\ Laboratorio de Ecología Animal, Centro Interdisciplinario de Investigación para el Desarrollo Integral Regional, Unidad Oaxaca, Instituto Politécnico \\ Nacional. Hornos 1003, 71230 Santa Cruz Xoxocotlán Oaxaca, México. \\ \asantosm90@hotmail.com
}

\begin{abstract}
Resumen. Se estimaron los movimientos y el área de actividad del ratón Liomys irroratus en una selva mediana del sureste del estado de Oaxaca, México. Los machos presentaron áreas de actividad más grandes que las hembras (126 $\mathrm{m}^{2}$ vs. 21.1); asimismo, en la temporada seca las áreas fueron mayores que en la de lluvias, pero en este caso las diferencias no fueron estadísticamente significativas. El tamaño promedio anual de área de actividad para todos los individuos de la población se estimó en $87.48 \mathrm{~m}^{2}$. Los machos no presentaron superposición de sus áreas de actividad con otros machos, y fue baja tanto entre hembras como entre individuos de sexos diferentes. Los machos tendieron a recorrer distancias promedio más grandes que las hembras $(69.31 \mathrm{~m} v \mathrm{~s}$. 33). En la temporada de lluvias los individuos se movieron significativamente más que en la seca (58.12 m vs. 44.19). El tamaño de las áreas de actividad de las hembras mostró una correlación estadísticamente significativa e inversa con el número de árboles y el de arbustos, y en estas áreas el número de oquedades y las pendientes son mayores que en las de los machos. Los resultados permiten suponer que la especie tiene un sistema de apareamiento de poligamia, manteniendo su territorio hasta que inicia la temporada reproductiva.
\end{abstract}

Palabras clave: Heteromyidae, territorialidad, área de actividad.

\begin{abstract}
We estimated the movements and activity area of the spiny pocket-mice Liomys irroratus in a semideciduos tropical forest at the southeastern of the state of Oaxaca, Mexico. Males have larger activity areas than females (126 $\mathrm{m}^{2}$ vs. 21.1), also, in the rainy season these were higher than in the dry season, but in this case the differences were not statistically significant. The annual average size of the activity area for all individuals in the population was of 87.48 $\mathrm{m}^{2}$. Males showed no overlap in their activity areas with other males, while between females and between members of different sexes this was low. Males showed average travel distances larger than females $(69.31 \mathrm{~m} \mathrm{vs.} \mathrm{33).} \mathrm{In} \mathrm{the}$ rainy season individuals moved significantly more than in the dry season (58.12 $\mathrm{m} v \mathrm{~s}$. 44.19). The size of activity areas of the females showed a significant and inverse correlation with the number of trees and shrubs, and these areas had higher number of cavities and greater slope that those occupied by males. The results suggest that the species has a polygamous breeding system, where the territory is held until the beginning of the breeding season.
\end{abstract}

Key words: Heteromyidae, territoriality, activity area.

\section{Introducción}

El área de actividad se define como el sitio donde un animal normalmente realiza sus actividades rutinarias (Burt, 1943). En esta definición se encuentra implícita la capacidad de sostén que tiene dicha área para los individuos que la habitan (Laver, 2005). El tamaño promedio del área de actividad y el grado de superposición entre los individuos pueden variar entre poblaciones de una especie (McLoughlin et al., 2000). Estas características están influidas por varios fac-

Recibido: 07 abril 2011; aceptado: 07 febrero 2012 tores, entre los que destacan los hábitos alimenticios, talla del animal y necesidades metabólicas (Gittleman y Harvey, 1982), la calidad del hábitat, la distribución y abundancia del alimento, la densidad poblacional, el sexo, la edad, la condición social, la actividad reproductiva (Corbalán y Ojeda, 2005), los regímenes de humedad y la depredación (Ostfeld, 1985, 1990). De éstos, el más prominente probablemente sea la disponibilidad de alimento (Boutin, 1990). Las diferencias en el tamaño del área de actividad entre sexos pueden estar relacionadas con las estrategias reproductivas y los sistemas de apareamientos (Gentile et al., 1997).

El estudio del área de actividad que ocupan los individuos de las especies animales suele ser importante, ya que 
el tamaño del área que utilizan, así como la frecuencia y la lejanía con que se mueven son indicadores importantes de sus requerimientos de recursos (Plummer y Congdon, 1994) así como de la magnitud espacial de sus interacciones con individuos coespecíficos o con individuos de otras especies.

Esta información es particularmente relevante cuando se trata de especies consideradas como componentes clave en los procesos de sucesión y regeneración de las selvas tropicales (De Mattia et al., 2004, 2006), como es el caso de algunas especies de pequeños roedores heterómidos que dispersan plantas pioneras hacia los sitios perturbados y sus alrededores (Cimé-Pool et al., 2010).

Los miembros de la familia Heteromyidae representada por los géneros Dipodomys, Chaetodipus, Perognathus, Heteromys y Liomys poseen abazones externos en las mejillas que utilizan para almacenar alimentos. Esta adaptación les permite la recolección eficiente y el transporte de alimentos en periodos cortos (Reichman, 1983), así como su almacenamiento en galerías construidas bajo troncos, rocas y arbustos (Espinosa y Chávez, 2005), capacitándolos como depredadores/removedores importantes de semillas, lo cual influye en la estructuración de comunidades de plantas anuales en hábitat desérticos (Sánchez-Cordero y Fleming, 1993) y posiblemente en otras selvas en las que este tipo de roedores se encuentran presentes (Howe y Smallwood, 1982; Sánchez-Rojas et al., 2004).

El presente estudio enfocado a L. irroratus (Heteromyidae) tuvo como objetivo general realizar un acercamiento al conocimiento de las interacciones entre los individuos y el ambiente, considerando la importancia biológica de la especie dentro de su hábitat, y ante el desconocimiento de éstos aspectos etológicos de la biología de la especie.

\section{Materiales y métodos}

El estudio se realizó en el ejido Cerro Tepezcuintle (1808'51" N, 96²0’28" O), en el municipio de San Miguel Soyaltepec, ubicado en la cuenca del río Papaloapan, en el estado de Oaxaca, México (Fig. 1). Presenta un clima cálido subhúmedo, con temperatura promedio de $25.5^{\circ} \mathrm{C}$ y precipitación pluvial promedio de $2790.1 \mathrm{~mm}$, siendo los meses de mayo a octubre la temporada lluviosa, y de noviembre a abril la seca (INEGI, 2007). El tipo de vegetación es selva mediana en el que predominan géneros como Enterolobium, Astronium, Ficus, Cordia, Manilkara, Cedrela, Bursera, Cecropia, Asplenium, Vriesea, Alsophuila, Polypodium, Desmoncus, Dioscorea, Philodendron y Syngonium (Rzedowski, 1994; Challenger, 1998). El tipo de suelo es luvisol y rendzina, caracterizado por ser pedregoso, con un gran número de pequeñas cuevas y oquedades que sirven como refugios a algunas especies de mamíferos pequeños.

Se efectuaron visitas mensuales de 5 días de duración de julio del 2007 a julio del 2008. Se trazaron 2 cuadros de 1.1025 ha, cada uno. En cada cuadro se colocaron 64 trampas Sherman $(7.5 \times 9.0 \times 30 \mathrm{~cm})$ arregladas en 8 columnas y 8 filas, separadas por $15 \mathrm{~m}$ cada una. Las trampas se revisaron y recebaron diariamente (en caso necesario) con una mezcla de semillas de girasol con vainilla, colocando menos de 10 semillas para evitar efectos de trampofilia (Menkens y Anderson, 1988).

Se empleó el método de captura-marcaje-liberaciónrecaptura (Krebs, 1999) para la identificación individual de los organismos por medio de grapas para oreja de numeración consecutiva, tipo 1005-1 (National Band \& Tag Co., EUA). Posteriormente, se registraron los siguientes datos: sexo, peso (en gramos), edad (adulto o joven) y condición reproductiva (hembras preñadas e individuos activos, aquellos con evidencias, como glándulas mamarias desarrolladas en las hembras, y testículos escrotados en el caso de los machos, o en caso contrario, inactivo), así como la trampa (ubicación) de la captura o recaptura. Después de tomar los datos, los organismos se liberaron en el mismo lugar de la captura.

Para la caracterización del área de actividad se consideraron únicamente los sitios en que se capturaron individuos residentes, i.e., aquellos recapturados al menos en 3 meses durante el estudio (Hernández, 2003). Cada sitio se consideró como el centro de un círculo de $1 \mathrm{~m}$ de radio, que determinó un área $3.14 \mathrm{~m}^{2}$ (García et al., 2002), en esta área se contó el número de árboles y arbustos, el de oquedades formadas por las rocas, troncos caídos y la pendiente. Estas variables fueron elegidas porque las propiedades del sustrato y la vegetación son factores físicos que afectan directamente a los roedores por medio de su influencia sobre las madrigueras, locomoción y forrajeo en ambientes templados (Brown y Harney, 1993), pues se carece de información al respecto en zonas tropicales.

El área de actividad se calculó con el método del polígono mínimo convexo (Hayne, 1949); debido al número bajo de datos obtenidos, se excluyó el 5\% de las observaciones extremas. Para el análisis del movimiento se consideró la distancia promedio recorrida, para lo cual se realizó la sumatoria de los desplazamientos lineales que los organismos (hembras o machos) efectuaron entre sitios de trampeo a lo largo de las temporadas del año (lluvia o sequía) dividido entre el número de desplazamientos. Las áreas y las distancias se compararon entre sexos y temporadas por medio de un análisis de varianza no paramétrico, la prueba de Kruskal-Wallis, ajustada para empates en los rangos de los valores. 
Debido al número bajo de recapturas, se consideró más apropiado calcular la superposición de los sitios de captura que la de áreas (Corbalán y Ojeda, 2005), por medio del índice de superposición de O’Farrell (1980):

$$
I S=M_{i} \cap M_{j} / M_{i t o t}
$$

donde $M_{i} \cap M_{j}=$ número de trampas que el grupo $i$ (sexo, edad, etc.) comparte con los individuos del grupo $j$ en un periodo, temporada o año según sea el caso; $M_{i \text { total }}=$ número total de sitios de captura para el grupo $i$ (sexo o temporada). El valor de este índice se multiplicó por 100 para expresar el grado de superposición como porcentaje.

Con la finalidad de caracterizar las áreas de actividad, se realizó un análisis de componentes principales, extraídos de una matriz de correlación con los datos estandarizados para observar si existió alguna afinidad ecológica por sexo en las áreas de actividad utilizadas por los individuos, así como identificar las variables físicas que permiten esta diferenciación. El grado y sentido de la relación entre cada una de las variables y el tamaño del área de actividad se estimó por medio del coeficiente de correlación de Pearson.

\section{Resultados}

El esfuerzo de captura total fue de 8320 nochestrampa, con el que se obtuvieron 58 localizaciones de 21 individuos adultos que se consideraron residentes en este trabajo. Los machos presentaron áreas de actividad significativamente más grandes que las de las hembras, así mismo, en la temporada lluviosa éstas son mayores que en la seca, pero en este caso las diferencias no son estadísticamente significativas, la interacción entre sexo y temporada fue significativa (Cuadro 1). Dados estos resultados, se estimó el valor promedio de área de actividad para todos los individuos de la población en $87.48 \mathrm{~m}^{2}$ (D. E.=157.67). Los machos no presentaron superposición de sus áreas de actividad con otros machos $\left(I S_{\text {Machos }}=0\right)$, mientras que entre hembras fue del $14.8 \%$, porcentaje similar al obtenido de la superposición entre miembros de sexos diferentes $\left(I S_{\text {Machos- }}\right.$ Hembras $=16.66 \%$ ). En la temporada lluviosa, los individuos de la población (hembras y machos) presentaron el 7.14\% de superposición en las áreas de actividad, y en la temporada de sequía fue del $15.38 \%$.

En cuanto a las distancias promedio, los machos recorrieron distancias significativamente más grandes que las hembras. En la temporada de lluvias los individuos se mueven significativamente más que en la seca. Con base en estos resultados se estimó el promedio de distancias en la temporada de lluvia en $58 \mathrm{~m}$ y en la seca 44. La interacción entre sexo y temporada no fue significativa (Cuadro 1).
Para caracterizar las áreas de actividad se consideraron 24 sitios de captura, de 13 hembras y 11 machos residentes. Los 2 primeros componentes principales explicaron el $62.51 \%$ de la varianza total (Cuadro 2), pero sólo el segundo parece estar relacionado con diferencias en los atributos de los sitios de captura entre machos y hembras (Fig. 2) y tiene asociado el $27.78 \%$ de la varianza. Lo sitios ocupados por las hembras se caracterizaron porque es más el número de oquedades y la pendiente es mayor que en los ocupados por los machos. El tamaño de las áreas de actividad de las hembras mostró una correlación estadísticamente significativa e inversa con el número de árboles y el de arbustos, mientras ninguna de las 4 variables mostró correlación con el tamaño del área de los machos (Cuadro 2).

\section{Discusión}

Aunque no existen estudios previos en la zona que documenten el tamaño poblacional de L. irroratus, tanto el número de individuos registrados como el de recapturas parecen bajos, si se consideran los tamaños de las muestras observados en otros sitios con esfuerzos de colecta similares e incluso menores al aplicado en este estudio. Por ejemplo, en el sureste del estado de Morelos se capturaron 93 individuos con 8800 noches-trampa (García et al., 2002) y en el altiplano potosino 527 individuos con 5400 noches-trampa (Mellink, 1995). Una posible explicación es que el año de muestreo fue especialmente seco; de acuerdo con los registros de la Estación Climatológica Ojitlán (18³'48.7" N, 96²3’4.9” O) de la Red Nacional de Estaciones Estatales Agroclimatológicas del Instituto Nacional de Investigaciones Forestales, Agrícolas y Pecuarias, durante la temporada seca de enero a mayo de 2007, precisamente antes de iniciar el estudio, sólo se registraron $74.1 \mathrm{~mm}$ de precipitación y el total anual fue de $349 \mathrm{~mm}$, mientras que en 2008 se registraron 295 y 2 569.4 respectivamente. La disminución en la precipitación causó una disminución en la cobertura herbácea, factor importante para los organismos debido a que les provee de refugio, protección y alimentación. Esta relación, especialmente fuerte entre precipitación y el tamaño poblacional de L. irroratus, ya ha sido observada, por ejemplo, en el altiplano potosino (Mellink, 1995), incluso se sabe que la especie no está presente en áreas del centro-norte del Altiplano mexicano que reciben menos de $500 \mathrm{~mm}$ de precipitación anual (Dowler y Genoways, 1978).

El tamaño del área de actividad promedio de L. irroratus en Cerro Tepezcuintle es similar a lo registrado en otros estudios (e.g., $221.15 \mathrm{~m}^{2}$ en el estado de Morelos, GarcíaEstrada 1999) y la tendencia de los machos a ocupar áreas más grandes que las hembras está presente en otras espe- 
Cuadro 1. Comparación de los valores promedio del tamaño de las áreas de actividad (en $\mathrm{m}^{2}$, desviación estándar entre paréntesis) y distancias promedio recorridas (en m) por Liomys irroratus, por sexo, temporada y su interacción

\begin{tabular}{|c|c|c|c|c|c|c|}
\hline \multicolumn{7}{|c|}{ Áreas } \\
\hline Fuente de variación & & $N$ & Media $(D E)$ & $H$ & g. $l$. & $P$ \\
\hline \multirow{2}{*}{ Sexo } & Hembras & 6 & $21.10(28.89)$ & 5.789474 & 1 & 0.016 \\
\hline & Machos & 6 & $126(51.86)$ & & & \\
\hline \multirow[t]{2}{*}{ Temporada } & Lluvias & 7 & $108.41(47.82)$ & 0.006616 & 1 & 0.935 \\
\hline & Seca & 5 & $38.69(34.5)$ & & & \\
\hline \multirow[t]{4}{*}{ Sexo, temporada } & Hembras, lluvias & 3 & $7.19(6.62)$ & 88.56 & 3 & 00.035 \\
\hline & Hembras, sequía & 3 & $34.99(8.31)$ & & & \\
\hline & Machos, lluvias & 4 & $209.62(245.2)$ & & & \\
\hline & Machos, sequía & 2 & $42.39(37.6)$ & & & \\
\hline \multicolumn{7}{|c|}{ Distancias } \\
\hline \multirow[t]{2}{*}{ Sexo } & Hembras & 18 & $33(16.64)$ & 4.941 & 1 & 0.0262 \\
\hline & Machos & 12 & $69.31(209.38)$ & & & \\
\hline \multirow[t]{2}{*}{ Temporada } & Lluvias & 16 & $58.12(204.46)$ & 3.97 & 1 & 0.0461 \\
\hline & Seca & 14 & $44.19(20.11)$ & & & \\
\hline \multirow[t]{4}{*}{ Sexo, temporada } & Hembras, lluvias & 6 & $40.20(40.09)$ & 6.022 & 3 & 0.11 \\
\hline & Hembras, sequía & 12 & $25.79(21.98)$ & & & \\
\hline & Machos, lluvias & 10 & $76(48.81)$ & & & \\
\hline & Machos, sequía & 2 & $62.59(88.51)$ & & & \\
\hline
\end{tabular}

N, tamaño muestra; H, valor del estadístico de Kruskal-Wallis; g. 1, grados de libertad; P, valor de significancia.

Cuadro 2. Vectores y valores eigen de los 2 primeros componentes principales (CP1 y CP2), valor promedio (desviación estándar entre paréntesis) y correlación (cuando fue significativa en negritas, significancia entre paréntesis) de 4 variables con el tamaño del área de actividad de machos y hembras residentes de Liomys irroratus

\begin{tabular}{|c|c|c|c|c|c|c|}
\hline \multirow{3}{*}{ Variable } & \multicolumn{2}{|c|}{ Eigen vectores } & \multicolumn{4}{|c|}{ Sexo } \\
\hline & \multirow[b]{2}{*}{$C P 1$} & \multirow[b]{2}{*}{$C P 2$} & \multicolumn{2}{|c|}{ Hembras } & \multicolumn{2}{|c|}{ Machos } \\
\hline & & & Valor promedio & Correlación & Valor promedio & Correlación \\
\hline Número de árboles & -0.653 & 0.235 & $2.214(1.22)$ & $-0.856(0.00144)$ & $3.312(0.68)$ & $-0.286(0.7005)$ \\
\hline Número de arbustos & 0.64 & 0.2116 & $5.67(4.26)$ & $-0.729(0.02810)$ & $6.29(1.27)$ & $0.115(0.78663)$ \\
\hline Número de oquedades & -0.32 & -0.612 & $3.607(1.31)$ & $-0.329(0.62425)$ & $2.1(1.3)$ & $0.257(0.72298)$ \\
\hline Pendiente & 0.245 & -0.724 & $42.5(15)$ & $-0.254(0.70757)$ & $34.47(15)$ & $0.558(0.27697)$ \\
\hline Eigen valor & 1.389 & 1.1111 & -- & & - - & \\
\hline$\%$ varianza explicada & 34.73 & 27.8 & -- & & - & \\
\hline $\begin{array}{l}\% \text { varianza explicada } \\
\text { acumulada }\end{array}$ & 34.73 & 62.53 & -- & & -- & \\
\hline
\end{tabular}

cies de heterómidos como Liomys pictus (Sánchez-Rojas et al., 1994) y Heteromys gaumeri (Hernández Betancourt et al., 2003).

El uso de áreas mayores en la temporada seca que en la lluviosa por parte de las hembras puede ser explicado considerando su actividad reproductiva y la producción de semillas en la zona. Aunque se registraron individuos con evidencias de actividad reproductiva a lo largo de todo el año, éstos predominaron al final de la temporada de sequía y principio de la temporada lluviosa. Durante este periodo, las hembras que se encuentran preñadas o en lactancia exhiben una gran demanda energética (Millar, 1975) y por lo tanto requieren economizar energía para asegurar la supervivencia de los neonatos (Ribble y Stanley, 1998; Bond y Wolf, 1999). En el caso de los machos, en la temporada lluviosa invierten la mayor cantidad de energía en la recolección y almacenamiento de semillas (Brown y Harney, 1993), lo que probablemente se refleja en un área de actividad mayor. Las semillas almacenadas se utilizan en periodos de baja disponibilidad de alimento, como es la temporada seca. Esta capacidad de consumir alimentos almacenados, junto con el aletargamiento, permite que durante la temporada seca aumente su supervivencia (Brown y Harney, 1993) y disminuya el área de actividad.

El que los machos no muestren superposición de áreas de actividad con miembros del mismo sexo permite supo- 


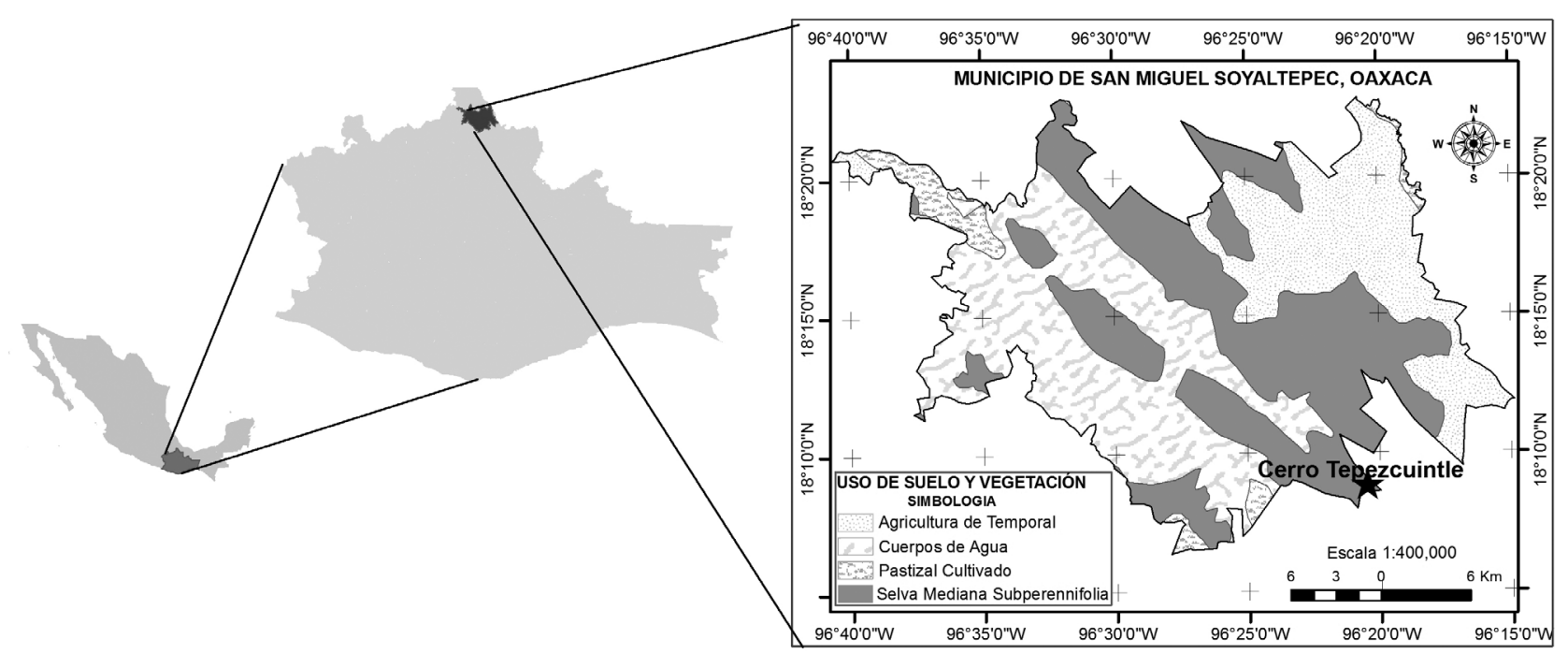

Figura 1. Ubicación geográfica de Cerro Tepezcuintle.

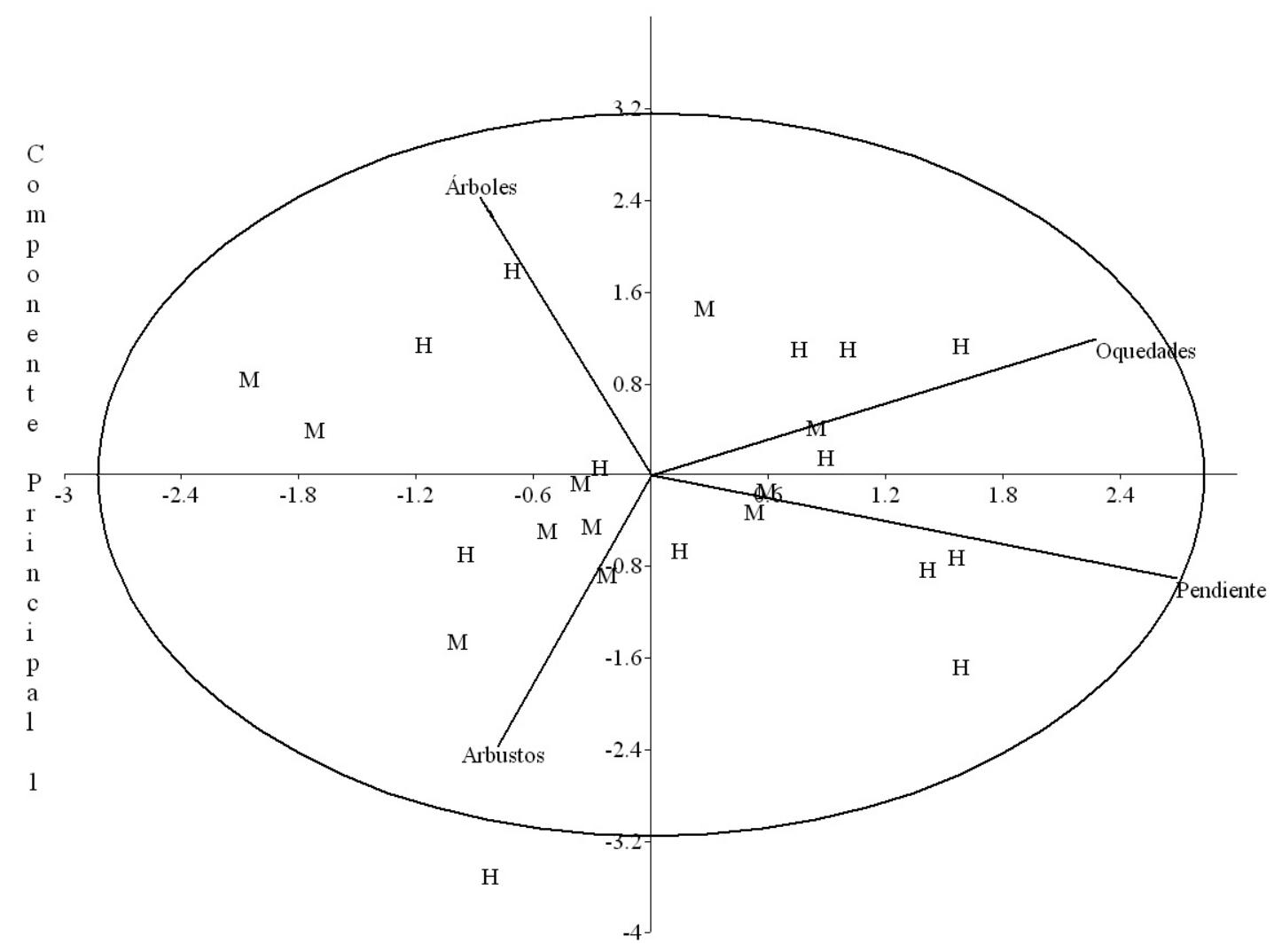

Componente Principal 2

Figura 2. Proyección de las áreas de actividad de 13 hembras (H) y 11 machos (M) de Liomys irroratus en los 2 primeros componentes principales, la elipse incluye el 95\% de las observaciones. Se muestran superpuestos los eigen vectores de las 4 variables originales. 
ner que son organismos territoriales. Del mismo modo, la baja superposición entre hembras (14.8\%) confirma la existencia de territorialidad marcada en los roedores del género Liomys, por lo menos durante la época reproductiva (Fleming, 1974). En cuanto a la superposición entre machos y hembras, se observó que las hembras presentan cierta tolerancia hacia los machos $(16.66 \%)$, probablemente debido a la reproducción. En cuanto a la variación entre temporadas, se observó menor superposición de las áreas en la estación de lluvia (7.14\%) que en la seca (15.38\%), lo cual pudo estar determinado por la existencia de una mayor producción de semillas en la temporada lluviosa, que limita la necesidad de superposición de áreas con otros individuos en zonas ricas en alimento. Por otra parte, existe la posibilidad de que la baja superposición espacial en los muestreos de invierno y verano simplemente puede reflejar la baja densidad poblacional (y la consecuente carencia de datos de captura) y no selección de microhábitat (Corbalán y Ojeda, 2005).

Las áreas de actividad de las hembras tienen un mayor número de oquedades y mayor pendiente, lo que puede explicarse considerando que estas características representan una mayor protección tanto para ellas como para sus crías. En el caso de los machos, no se observó ningún patrón de correlación, ello debido a que se considera que las áreas de actividad de los machos no están esencialmente condicionados por el hábitat, sino por los movimientos de las hembras, dado por las actividades reproductivas. (Emlen y Oring, 1977; Sánchez-Cordero y Fleming, 1993).

En cuanto a la distancia promedio recorrida para cada temporada del año, se observaron mayores movimientos en la temporada de lluvia que en la temporada de sequía, de manera similar a los observados en el sureste de Morelos (García et al., 2002), pero de menor magnitud. Es probable que se deba a la actividad reproductiva que se prolonga hasta inicio de la temporada de lluvias. Por otro lado, estas diferencias pueden estar relacionadas con la capacidad de almacenamiento de los recursos alimenticios (Fleming, 1974; Espinosa y Chávez, 2005) o con la cobertura que presenta la zona, ya que en la temporada de sequía la vegetación es poca, lo que resulta en una baja cobertura por parte de árboles y arbustos, y en consecuencia son más evidentes a los depredadores (Fleming, 1974). La mayor movilidad de los machos se ha observado en otras especies, como L. pictus, e incluso se ha propuesto que la dispersión predominantemente de los machos y la filopatría de las hembras son elementos característicos de la estructura social de la especie (Vázquez-Domínguez et al., 2002).

Las variaciones en los movimientos y en el tamaño del área de actividad que presentan los individuos, hacen suponer que L. irroratus presenta un sistema de apareamiento poligínico, manteniendo su territorio hasta la llegada de la temporada reproductiva, lo que se ve reforzado con la presencia de conductas territoriales por parte de los machos y la poca tolerancia social entre las hembras, que son características de especies polígamas. Las diferencias en las distancias recorridas, así como en el tamaño del área de actividad observada en este estudio, en comparación con otros heterómidos, parece estar relacionados con el grado de la estacionalidad de su hábitat, el cual influye principalmente en la producción de alimentos. Liomys irroratus parece seleccionar áreas de actividad mostrando cierta agrupación por sexos, lo cual apoya la idea de que la selección de sitios por parte de las hembras está determinada principalmente por la disponibilidad de alimento y refugio, mientras que la ubicación de los sitios de actividad y los desplazamientos de los machos parecen estar determinados por la presencia de las hembras.

\section{Agradecimientos}

La comunidad del ejido Cerro Tepezcuintle, Oaxaca proporcionó facilidades para realizar el estudio. El Consejo Nacional de Ciencia y Tecnología de México proporcionó una beca a Aremi E. Santiago-Marcial para estudios de posgrado y la Subdirección de Investigación y Posgrado del Instituto Politécnico, el financiamiento para la ejecución del proyecto (apoyos SIP-20070826 y SIP-20080431 a AS-M).

\section{Literatura citada}

Bond, M. L. y O. J. Wolff. 1999. Does access to female or competition among males limit male home range size in a promiscuous rodent Journal of Mammalogy 80:1243-1250.

Boutin, S. 1990. Food supplementation experiments with terrestrial vertebrates: patterns, problems, and the future. Canadian Journal of Zoology 68:203-220.

Brown, J. H. y B. A. Harney. 1993. Population and community ecology of heteromyid rodents in temperate habitats. In Biology of the Heteromyidae. Special Publications 10, H. H. Genoways y J. H. Brown (eds.). American Society of Mammalogysts. p. 618-650.

Burt, W. H. 1943. Territoriality and home range concept as applied to mammals. Journal of Mammalogy 24:346-352.

Cimé-Pool, J.A., S. F. Hernández-Betancourt, R. C. Barrientos, A. A. Castro-Luna. 2010. Diversidad de pequeños roedores en una selva baja caducifolia espinosa del noreste de Yucatán, México. Therya 1:23-40.

Corbalán, V. E. y R. A. Ojeda. 2005. Home range of a rodent population in the Monte desert (Mendoza, Argentina). Mastozoología neotropical 12:145-152. 
DeMattia, E. H., L. M. Curran, y B. J. Rathcke. 2004. Effect of small rodents and large mammal on neotropical seeds. Ecology 85:2161-2170.

DeMattia, E. A., B. J. Rathcke, L. M. Curra, R. Aguilar, y O. Vargas. 2006. Effect of small rodent and large mammals exclusion on seedling recruitment in Costa Rica. Biotropica 38:196-202.

Dowler, R. C. y H. H. Genoways. 1978. Liomys irroratus. Mammalian Species 82:1-6.

Emlen, S. T. y L. W. Oring. 1977. Ecology, sexual selection, and evolution of mating systems. Science 197:215-223.

Espinosa, L. A. y T. C. Chávez. 2005. Liomys irroratus. Ratón espinoso. In Los mamíferos silvestres de México, G. Ceballos y G. Oliva (eds.). Comisión Nacional para el Conocimiento y Uso de la Biodiversidad/ Fondo de Cultura Económica. México, D. F. p. 628-629.

Fleming, T. H. 1974. Social organization in two species of Costa Rican heteromyid rodents. Journal of Mammalogy 55:543-56.

Foster, R. B. 1982. Famine on Barro Colorado Island. In Ecology of a tropical forest: seasonal rhythms and long-term changes, E. G. Leigh Jr., A. S. Rand y D. M. Windsor (eds.). Smithsonian Institution Press, Washington, D. C. p. 201-212.

García, C. 1999. Estudio en dos comunidades de roedores en dos áreas con diferente grado de alteración en el sureste de Morelos. Tesis Maestría, Facultad de Ciencias, Universidad Nacional Autónoma de México, México, D. F. 107 p.

García, C., M. L. Romero y C. Sánchez. 2002. Comparison of rodent communities in sites with different degrees of disturbance in deciduous forest of southeastern Morelos, Mexico. Acta Zoológica Mexicana (nueva serie) 85:153-168.

Gittleman, J. L. y P. H. Harvey. 1982. Carnivore home range size metabolic needs ecology. Behavior and Sociobiology 10:57-63.

Hayne, D. W. 1949. Calculation of size of home range. Journal of Mammalogy 30:1-18.

Hernández Betancourt, S., R. López-Wilchis, J. A. Cimé Pool y S. Medina Peralta. 2003. Área de actividad, movimiento y organización social de Heteromys gaumeri Allen y Chapman, 1897 (Rodentia: Heteromyidae) en una selva mediana subcaducifolia de Yucatán, México. Acta Zoológica Mexicana (nueva serie) 90:77-91.

Krebs, C. J. 1999. Ecological methodology, segunda edición. Benjamin/Cummings, Menlo Park, California. 620 p.

Laver, P. N. 2005. Cheetah of the Serengeti plains: a home range analysis. Tesis, Maestría en Ciencias, Virginia Polytechnic Institute and State University, Blacksburg, Virginia. $135 \mathrm{p}$.

McLoughlin, P. D., T. E. Ferguson y T. E. Messier. 2000.
Intraspecific variation in home range overlap with habitat quality: a comparison among brown bear populations. Evolutionary Ecology 14:39-60.

Mellink, E. 1995. Uso de hábitat, dinámica poblacional y estacionalidad reproductiva de roedores en el altiplano potosino, México. Revista Mexicana de Mastozoología $1: 1-8$.

Menkens, G. E., Jr. y S. H. Anderson. 1988. Estimation of small mammal population size. Ecology 69:1952-1959.

Millar, J. S. 1975. Tactics of energy partitioning in breeding Peromyscus. Canadian Journal of Zoology 53:267-276.

Ostfeld, R. S. 1985. Limiting resources and territoriality in microtine rodents. American Naturalists 126:1-15.

Ostfeld, R. S. e I. I. Klosterman. 1990. Microtine social systems, adaptation and the comparative method. In Social system and population cycles in voles, R. H. Tamarin, R. S. Ostfeld, S. R. Pugh y G. Bujalska (eds.) Birkhauser, Berlin. p. 35-44.

Plummer, M. V. y J. D. Congdon. 1994. Radiotelemetric study of activity and movements of racers (Coluber constrictor) associated with a Carolina Bay in South Carolina. Copeia 1994:20-26.

Reichman, O. J. y M. V. Price. 1993. Ecological aspects of heteromyid foraging. In Biology of the Heteromyidae, Special publication 10, H. H. Genoways y J. H. Brown (eds.). American Society of Mammalogists. p. 539-574.

Ribble, D. O y S. Stanley. 1998. Home ranges and social organization of syntopic Peromyscus boylii and P. truei. Journal of Mammalogy 79:932-941.

Sánchez-Cordero, V. y T. H. Fleming. 1993. Ecology of tropical heteromyidae. In Biology of the heteromyidae. Special Publication 10, H. H. Genoways y J. H. Brown (eds.). American Society of Mammalogists. p. 596-617.

Sánchez-Rojas, G., M. A. Briones-Salas y V. Sánchez-Cordero. 1994. Área de actividad del ratón espinoso de abazones (Liomys pictus), en un BTC. Memoria del II Congreso Nacional de Mastozoología. AMMAC/ Universidad de Guadalajara/ Secretaría de Agricultura y Recursos Hidráulicos, Guadalajara, Jalisco. 170 p.

Sánchez-Rojas, G., M. Briones-Salas y V. Sánchez-Cordero. 2004. Effect of species, density and habitat on post-dispersal fruit and seed removal by spiny pocket mice in a tropical dry forest. Studies of Neotropical Fauna and Environment 39:1-6.

Vázquez-Domínguez, E., G. Ceballos y D. Piñero. 2002. Exploring the relation between genetic structure and habitat heterogeneity in the rodent Liomys pictus from Chamela, Jalisco. Acta Zoológica Mexicana (nueva serie) 86:17-28. 